AIAA-98-1755

\title{
RAPID MODELING WITH INNOVATIVE STRUCTURAL CONCEPTS
}

\author{
Max Blair* \\ Air Vehicles Directorate \\ Air Force Research Laboratory \\ WPAFB, OH 45433-7542
}

\author{
Stephen Hill II \\ TechnoSoft Inc. \\ Cincinnati, OH 45242-5545
}

\author{
Terrence A. Weisshaar $\dagger$ \\ Robert Taylor $\ddagger$ \\ Purdue University \\ West Lafayette, IN 47907-1282
}

\begin{abstract}
A proven general purpose design modeling environment is customized for use in the design of aircraft concepts. This effort complements other parallel efforts to develop manufacturing objects. This effort is one of a series of steps toward the goal of developing high fidelity design trades between cost and performance at the highest level.

Two factors make this work innovative. First, we are using an advanced design modeling environment with dependency tracking, demand-driven calculations and run-time object creation. Secondly, we are developing a structural modeling tool which addresses structural concepts at the earliest stage of design. This model uses independent global deformation functions. This removes the need for compatible multi-part mesh generation. The design turnaround time is reduced to the point that structural layout can be addressed at the conceptual level. This is accomplished with the transformation of triangular membrane elements from local to global coordinates.

The example focuses on the efficient transformation of triangular elements from local to global coordinates. This example includes maneuver control with static aeroelastic effects.
\end{abstract}

\section{TERMINOLOGY}

Dependency Tracking: Each model variable automatically tracks its influence on all other model variables and which variables quantities they influence.

Demand-Driven Calculations: Quantities are only calculated when they are needed. This is in contrast to serial programming, where the analysis proceeds according to a preprogrammed set of instructions.

* Research Aerospace Engineer, Senior AIAA Member

II Application Engineer, AIAA Member, ASME Member

$\dagger$ Professor, AIAA Fellow

\$ Graduate Student, Student AIAA Member

Adaptive Modeling Language is a trademark of TechnoSoft Inc.

All other company and product names are trademarks or registered trademarks of their respective owners

This paper is declared a work of the U.S. Government and is not subject to copyright protection in the United States.
Run-time Object Creation: Compiled rules and processes can be modified on-the-fly with interpretive code.

\section{INTRODUCTION}

The techniques described in this paper facilitate unconventional thinking. The envisioned process allows the designer to deviate from historical patterns and still maintain credibility.

A design process starts with mission requirements and accumulates data on past programs. Next, new technology is considered and the old requirements are examined again. The process continues until a convincing case can be made for an expensive development program. There is significant guesswork and intuition involved in this process which is based on conventional wisdom.

What happens when we design a system for which we have no database? For instance, the requirements for a Uninhabited Combat Air Vehicle (UCAV) will be significantly different from the requirements for current fighter systems (e.g. survivability, refueling, maneuver loads, maintenance etc.). If one is to realize novel concepts, the design process must rapidly assimilate high fidelity data. This is accomplished with the development of ready-to-use design objects at a low level which are driven from objects at a high level. This process is illustrated with the familiar systems engineering pyramid shown in Figure (1).

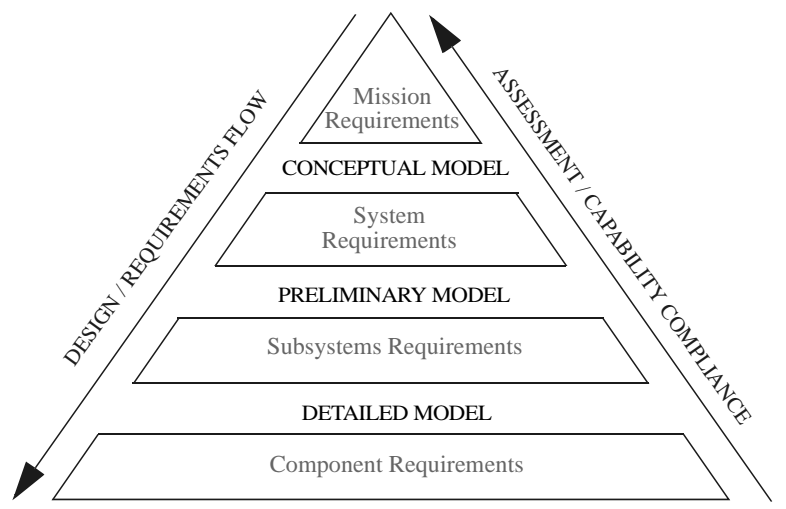

Figure (1) Systems Engineering Pyramid 
M. Blair, S. Hill, T. A. Weisshaar, R. Taylor,

Rapid Modeling with Innovative Structural Concepts

Integration is not just an issue for the flight system. An object-oriented environment with built-in dependencytracking, demand-driven calculations and run-time object creation significantly facilitates the integration and control of all aspects of a design.

While design integration is universally acclaimed, the motivation to actually invoke radical integration is not so easily attained. The requirement for design process integration has become clear after years of documented research experience.

In reference [1], a demonstration project was assembled in which a commercial geometric computer aided design code was used to parametrically control the geometry of the airframe outer surface and major substructures. Data was extracted with a series of configuration-dependent instructions and an aeroelastic optimization problem was accomplished for the set of parts. The process was practical for the purpose of resizing a specific configuration and structural geometry. It was not a good environment for design synthesis.

In reference [2], a design environment was used to retain and share data with two conceptual design codes and the model in reference [1]. Again, this design process was perhaps useful for resizing a design concept. Since the participating codes were developed independently, the process had elements of redundancy and inconsistency. Also the process was not tightly integrated and did not facilitate synthesis.

In reference [3], the same design environment in reference [2] was used to address preliminary structural weight and activity-based cost for use with a conceptual wing design study. This process facilitated integrated structural analysis.

In this paper, we address issues related to configuration design synthesis, again using the same design environment. In this process, design speed is important in early stages. In later stages, design fidelity is important. Higher fidelity design is demonstrated in reference [3]. Switching the process emphasis between speed and fidelity is very useful. This is possible only with a highly integrated design process. While we have not quite reached the point where we actually demonstrate data flow between levels of the design pyramid, the capability is clearly ready to be exploited.

\section{THE ADAPTIVE MODELING LANGUAGE}

The Adaptive Modeling Language ${ }^{\mathrm{TM}}$ (AML) environment which has evolved from a Materials Directorate (of the Air Force Research Laboratory) initiative in feature-based design to a commercial product in use by industries ranging from automotive, e.g., Ford Motor and Volvo; to aerospace, e.g., Lockheed-Martin, and
McDonnell-Douglas; and power generation, e.g., Zurn Balke-Durr and Siemens. AML incorporates a unique underlying object-oriented model for representing geometric and non-geometric features to support bi-directional constraint propagation across multiple design disciplines.

Consider the systems engineering pyramid. With automated dependency tracking, AML facilitates the control of a large number of design alternatives with a single set of driving requirements (feed forward). Dependency tracking can also be used to facilitate design parameterization (feed back). With demand-driven calculations, the designer can readily control when and how design information flows. AML already has built-in objects to address complex meshing and manufacturing issues. These capabilities, along with feature based geometry in a single open-access object-oriented environment make AML very attractive as a means of addressing complex air vehicle design integration.

\section{CONFIGURATION DESIGN SYNTHESIS}

Ideally, the design process begins with requirements which may be in the form of a mission profile, a flight envelope and so on. This is currently being addressed.

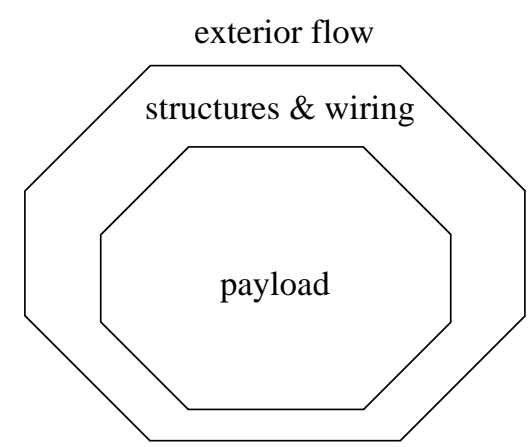

Figure (2) Nested Octagon Design Object

In Figure (2), we begin to capture the fuselage geometry with a nested octagon object. Octagons were used as a basic building element for this exploratory development. Nested octagons give us the ability to design with conflicting requirements. There are three types of dimensions, the inside boundary (payload, engines etc.), the exterior boundary (aerodynamic flow, signature etc.) and the space in between (structures, wiring, piping etc.). Only two of the three types can be independent. The third is dependent. The designer is allowed to switch the dependent dimension type, thus altering the order of design dependency between aerodynamics, structures and payload. This functionality is currently being captured in a graphical design interface. 
M. Blair, S. Hill, T. A. Weisshaar, R. Taylor,

Rapid Modeling with Innovative Structural Concepts

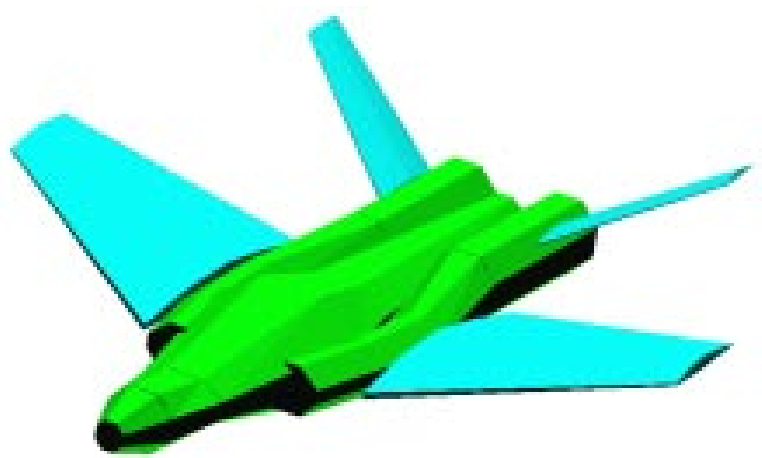

Figure (3) Conceptual Component Design of UCAV

AML design objects have been developed for components of an Uninhabited Combat Air Vehicle (UCAV). Some of these components are depicted in Figure (3). The weapons-bay and engine objects are depicted with interior and exterior octagonal prisms, thus capturing the subsystem and the exterior skin. The internal surface of the fuselage is depicted in Figure (4). The wing and tail objects are depicted with simple wing-like prisms. These prismatic objects resize rapidly. Later, a smooth surface will update efficiently with the demand-driven environment.

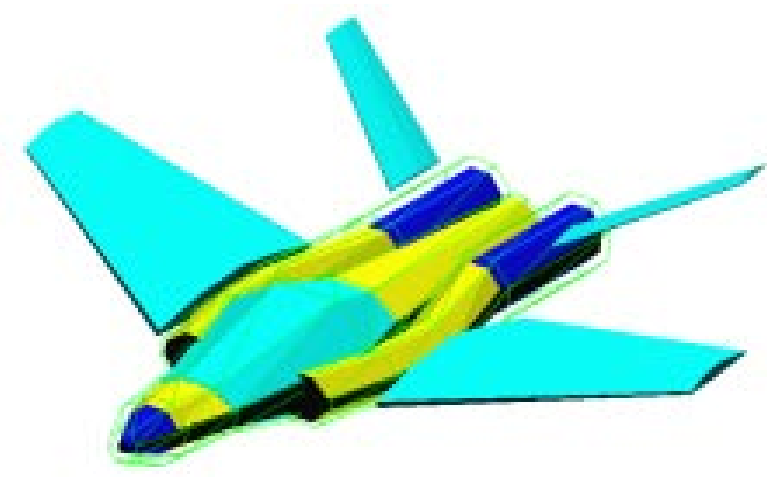

Figure (4) Internal Surface of UCAV Fuselage

These prismatic objects are useful for a coarse layout of the components. At this time, the fuselage model has only geometric attributes. The wing model has geometric, structural and aerodynamic attributes. The wing structures are based on equivalent plate theory (with shear deformation). Aerodynamic loads come from a simple vortex-lattice model. The main focus of this paper is to demonstrate how the structural parts can be designed rapidly.

The designer uses these objects to synthesize a configuration while developing and altering rules of depen- dency on-the-fly. The net result of developing the configuration and the dependencies together is an encoded version of the design process itself. For example, the designer can specify whether the engine moves with the weapons-bay or the weapons bay moves with the engine. In the future, the wings will be placed according to some weights and aerodynamic driven rules. Likewise for the fuel, duct, avionics, landing gear and so on.

\section{STRUCTURAL DESIGN SYNTHESIS}

The conceptual designer may elect to start looking at the structural layout for a number of reasons. First, the structures drive the weight. Second, structural flexibility influences the lift and drag. Third, the structures provide something to hang subsystems on to.

Traditionally, this level of structural modeling is reserved for the preliminary level of design. However, with rapid computer response, the designer can look at the influence of radically different structural concepts on weight and maneuverability. This global level of modeling may provide the load requirements for the next level of detail at the part level. Alternatively, this free-form structural modeling can feed into a higher fidelity multidisciplinary design optimization model for finer tuning.

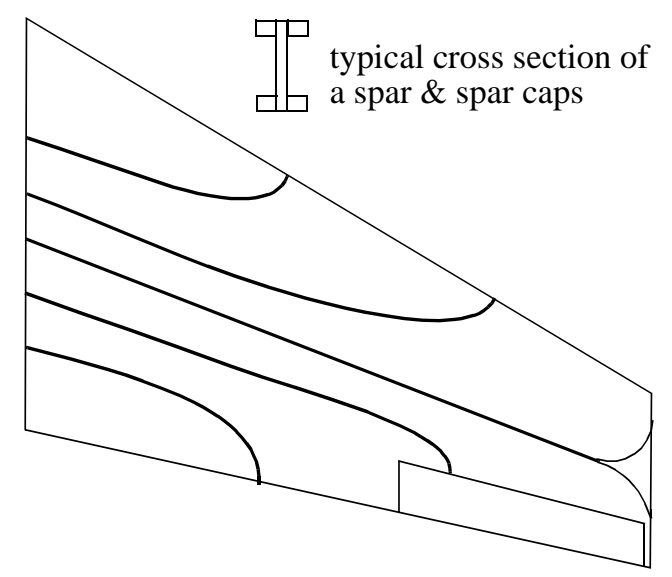

Figure (5) Organic Wing Substructure

The organic concept depicted in Figure (5) deviates from the rib-spar lattice-like pattern which most preliminary design tools seem to depend upon. This is a challenge for FEM because the mesh is difficult to generate without resorting to unstructured algorithms. FEM is also challenged because we have a difficult time identifying whether to model substructure with shear elements or membrane elements. More importantly, this organic concept may prove to be an affordable alternative 
M. Blair, S. Hill, T. A. Weisshaar, R. Taylor,

Rapid Modeling with Innovative Structural Concepts

because no ribs need to be constructed and assembled. The intersection between composite rib and spar parts is quite complex.

This organic concept is also depicted on the left wing of Figure (6). This model has traditional ribs and spars on the other lifting surfaces. Two different structures are provided on one figure in order to contrast the radical difference in wing design. Also shown on each wing and tail are the aerodynamic boxes on the mid-surface. The aerodynamic aspects will be discussed later.

The wing skin serves to pick up pressure loads and carry them to the spar caps. The spars pick up and channel the loads (e.g. point loads from a control surface or underwing store). The wing skin also serves to pick up on the shear stress which arises as the spars change direction.

In order to motivate further development, there is a need to rapidly model and explore such a concept at the conceptual level.

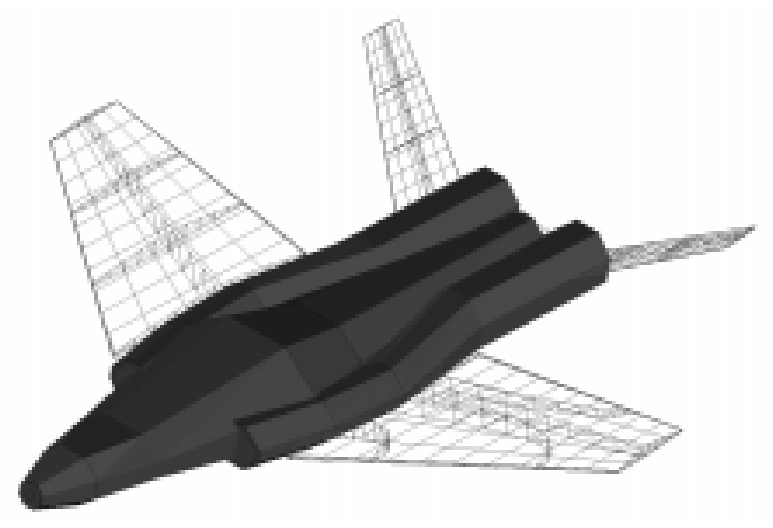

Figure (6) Lift Surface Sub-Structures for UCAV

\section{RAPID STRUCTURAL MODELING}

In the early days of aeroelastic tailoring [5], the TSO code [6] was developed in which wings and fuselage were rapidly modeled as an equivalent plate and beam. A plate structure assumes the existence of a neutral plane. By idealizing wing deformation with plate degrees of freedom, the neutral plane forces the user to assume the wing structure is symmetric with respect to this neutral plane.

The TSO code uses a traditional Rayleigh Ritz approach to analyze stress and strains based on Legendre polynomial functions in parametric space. Optimization is performed on a blended objective function and a suite of constraints involving aeroelastic loads and flutter.

Subsequent work by Giles uses equivalent plate theory to model segmented wings with cambered airfoils [7]. Later, Giles and Norwood created an hybrid structural model [8] with an equivalent plate wing and a finite element fuselage and pylon.

It makes sense that the deformation field should be retained independently from the solid model of the structure. The designer should be given the option to choose any set of kinematically admissible deformation functions. If an exact deformation solution is known, the designer should use that solution and a suite of others to form the set of deformation basis functions. Furthermore, these deformation functions should include shearing where substructure design is involved. This is an improvement over the assumption of a neutral plane in equivalent plate theory.

Given a basis for the deformation field, one can automate the integration of the strain energy over any solid geometry which represents structure.

The finite element method was coming into acceptance at the same time as TSO was being developed. With traditional FEM, the piecewise continuous deformation field is totally dependent on the mesh of elements. Each element is typically modeling with a set of low order "shape functions" and controlled at the corner grid points.

When new sub-structure is added, perhaps the entire FEM system may have to be re-meshed. This is because the nodes on the substructure have to line up with nodes on adjacent structure (e.g. exterior skin) in order for the piecewise continuous deformation field to make sense. Finite element meshing can be very time consuming, even prohibitive from the conceptual designer's point of view.

In a sense, we can look upon a Rayleigh Ritz model as a very high order finite element model. A wing may be coarsely segmented into fields of deformation. The deformation field could be a patchwork of $\mathrm{C}^{1}$ continuous parametric polynomial functions. For instance, the deformation for a segmented wing could be described with two Hermitian surface functions, one function for each of the two wing panels.

While referring to the variables in Figure (7), a basis set of deformation functions is given here for a single wing segment. Define the following quadratic shape functions for $0<\xi<1$

$$
\begin{aligned}
f_{0}(\xi) & =1-3 \xi+2 \xi^{2} \\
f_{1}(\xi) & =4 \xi-4 \xi^{2} \\
f_{2}(\xi) & =2 \xi^{2}-\xi
\end{aligned}
$$

These three shape functions correspond to the deformation at each of the three points, $\xi=(0,1 / 2,1)$ 
M. Blair, S. Hill, T. A. Weisshaar, R. Taylor, Rapid Modeling with Innovative Structural Concepts

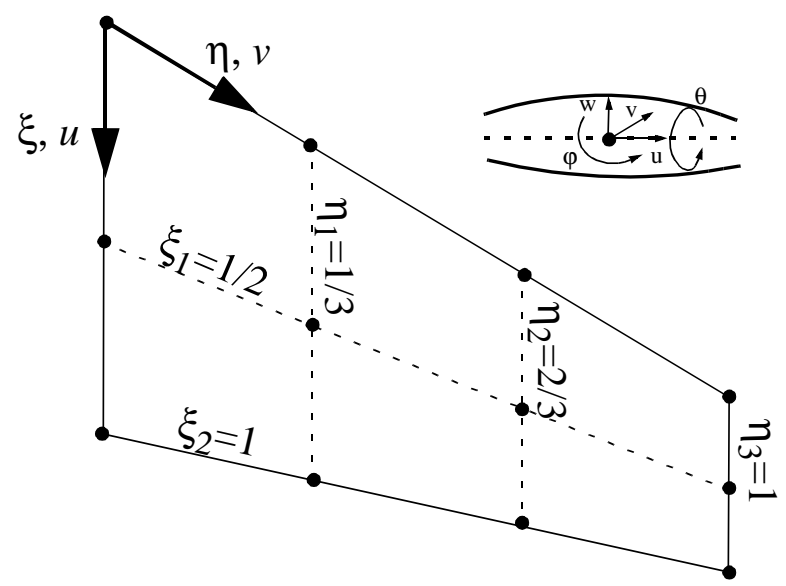

Figure (7) Parametric Wing Coordinates

Define the following cubic functions for $0<\eta<1$

$$
\begin{aligned}
& g_{0}(\eta)=-4.5 \eta^{3}+9 \eta^{2}-5.5 \eta+1 \\
& g_{1}(\eta)=13.5 \eta^{3}-22.5 \eta^{2}+9 \eta \\
& g_{2}(\eta)=-13.5 \eta^{3}+18 \eta^{2}-4.5 \eta \\
& g_{3}(\eta)=4.5 \eta^{3}-4.5 \eta^{2}+\eta
\end{aligned}
$$

These four shape functions correspond to the deformation at each of the four points, $\eta=(0,1 / 3,2 / 3,1)$

For a non-symmetric wing (with respect to the planform), there is a requirement to model in-plane deformation. The function $u(\xi, \eta)$ describes deformation in the $\xi$ direction

$$
u(\xi, \eta)=\sum_{i=0}^{2} f_{i}(\xi) \sum_{j=0}^{3} g_{j}(\eta) u\left(\xi_{i}, \eta_{j}\right)
$$

Here, 12 shape functions are created from combinations of equations (1) through (3) with equations (4) through (7). These 12 shape functions form the basis for all deformations in $u$.

The function $v(\xi, \eta)$ describes deformation in the $\eta$ direction

$$
v(\xi, \eta)=\sum_{i=0}^{2} f_{i}(\xi) \sum_{j=0}^{3} g_{j}(\eta) v\left(\xi_{i}, \eta_{j}\right)
$$

The function $w(\xi, \eta)$ describes out-of-plane deformation (in the direction normal to the local $\xi-\eta$ surface).

$$
w(\xi, \eta)=\sum_{i=0}^{2} f_{i}(\xi) \sum_{j=0}^{3} g_{j}(\eta) w\left(\xi_{i}, \eta_{j}\right)
$$

This is shown in Figure (8) with $w\left(\xi_{0}, \eta_{1}\right)=w\left(\xi_{1}, \eta_{1}\right)=$ $w\left(\xi_{2}, \eta_{1}\right)=1$. In order to capture shear deformation of the substructure shearing functions are introduced. Here, $\theta(\xi, \eta)$ is the rotation about the $\xi$ tangent.

$$
\theta(\xi, \eta)=\sum_{i=0}^{2} f_{i}(\xi) \sum_{j=0}^{3} g_{j}(\eta) \theta\left(\xi_{i}, \eta_{j}\right)
$$

Here, $\phi(\xi, \eta)$ is the rotation about the $\eta$ tangent.

$$
\phi(\xi, \eta)=\sum_{i=0}^{2} f_{i}(\xi) \sum_{j=0}^{3} g_{j}(\eta) \phi\left(\xi_{i}, \eta_{j}\right)
$$

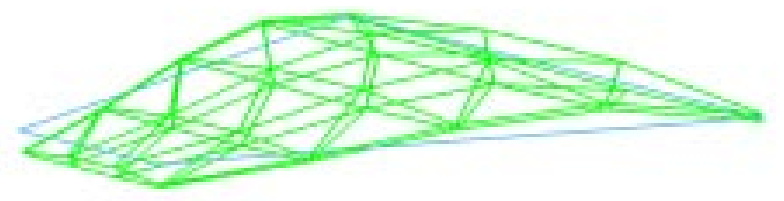

Figure (8) Example Deformation Function

Thus, the deformation field throughout the wing is controlled with 5 degrees of freedom $(u, v, w, \theta, \phi)$ at 12 control points for a total of 60 deformation controlling variables $\{q\}$. The corresponding 60 functions form the basis for the deformation field. If we cantilever the wing at $\eta=0$, then we reduce the set of basis functions from 60 to 45 .

Note that the choice of polynomial functions to model deformation is only a matter of convenience. If a higher fidelity FEM solution exists, that could be readily employed without polynomials. However, that capability has not yet been incorporated. This may require a FEM mesh generation capability which has built-in dependencies on the coarse mesh.

With a global deformation field, we do not have to create a compatible FEM mesh (between parts) which means we do not have to calculate geometric unions or intersections. This is potentially a large savings in computational time. The grid points along the boundaries of adjacent structural parts do not have to line up. The designer can simply lay out structure. Furthermore, integration of aerodynamic forces will be simplified. With a continuous deformation field, the designer does not have to redistribute load across a number of FEM grid points. It is a simple matter of integration weighted by the global deformation fields.

Now that we have a deformation field which is totally independent of any structural modeling, we have the freedom to select any structural modeling procedure. For instance, these deformations could serve as the basis for a Rayleigh Ritz analysis in which the deformation energy can be integrated over solid structures which the designer places into the deformation field. Alternatively, we could place finite elements in the deformation field, transforming from local to global coordinates. Here, we do the latter. 
M. Blair, S. Hill, T. A. Weisshaar, R. Taylor,

Rapid Modeling with Innovative Structural Concepts

Rather than integrate over solid geometry, it seems expedient to make use of existing finite element formulations to model structural stiffness and stress. The degrees-of-freedom for each element can be transformed from local to global coordinates. The advantage here is that grid points on one part do not have to line up with grid points on adjacent parts. Structural elements can be positioned anywhere because the set of global deformation functions is independently pre-determined.

This transformation from local element coordinates to global coordinates consumes some computational time but saves a considerable amount of designer time because there is no need to wait for a geometric union and a subsequent mesh over a suite of intersecting geometry. The resulting stiffness matrix will be precisely the same if we use traditional finite elements which have been constrained to global shapes after the traditional finite element mesh and stiffness have been formulated.

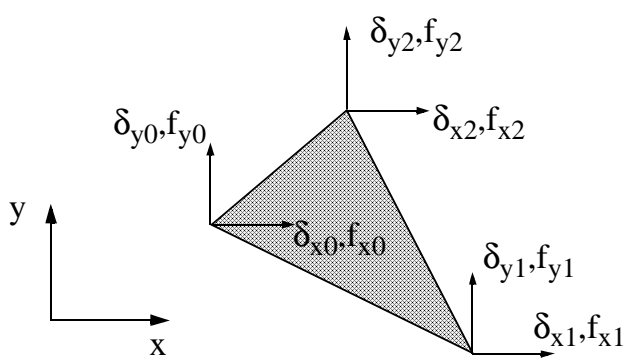

Figure (9) Local Triangular Membrane Coordinates

Membrane structures (two dimensional) are most easily employed. This is depicted in Figure (9). With the global deformation field, we do not have to be concerned with constraining out-of-plane degrees of freedom in the membrane. Still, in the end, we have to give stiffness to each of the 60 dof. By employing enough elements at their natural orientation, singularities are avoided. However, rather than risk singularities, we developed the option to superimpose a plate element on the membrane element for the wing skins. This way, we removed any possibility of a singularity in shear.

For a constant strain membrane triangle, we have six local degrees of freedom (dof).

$$
\{f\}=\left[k_{M}\right]\{\delta\}
$$

Rather than incorporating these six dof into a piecewise continuous set of local dof, we transform into the global set $\{q\}$ (45 dof)

$$
\{\delta\}=[T]\{q\}
$$

The $[T]$ is calculated as follows. Assume a unit amplitude for each global shape (e.g. a unit value for each $\left.\mathrm{u}\left(\xi_{\mathrm{i}}, \eta_{\mathrm{j}}\right)\right)$. Compute the three components of displacement at each of the three corners of the triangle for global shape $j$. At each corner $i$, take the dot product with each of the two unit local displacement vectors $\delta_{x i}$ and $\delta_{y i}$. Scale these two unit local displacement vectors by this product. These are the $(2 i, j)$ and $(2 i+1, j)$ elements of $[T]$. By repeating for each of the three corners, we fill out the $j^{\text {th }}$ column of $[T]$.

We elect to define the generalized (global) forces as

$$
\{Q\}=[T]^{T}\{f\}
$$

The system of six equations expands to 45 .

$$
\{Q\}=[K]\{q\}
$$

where

$$
[K]=[T]^{T}\left[k_{M}\right][T]
$$

The structural system is comprised of a number of parts, each of which are subdivided into a number of triangles. We compute an expanded $\left[K_{n}\right]$ matrix for each triangle and sum them. This forms the system stiffness matrix

$$
\left[K_{S}\right]=\sum_{n=0}^{m}\left[K_{n}\right]
$$

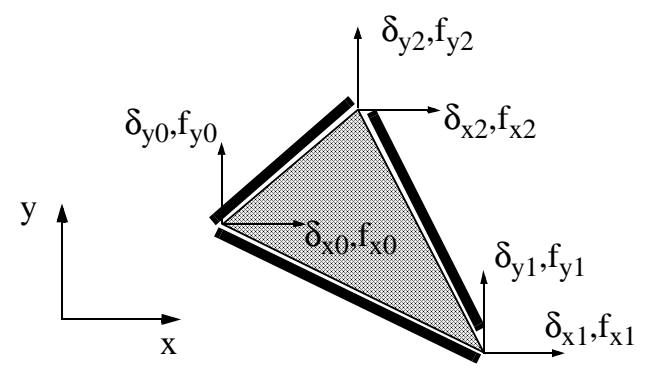

Figure (10) Reinforced Triangular Membrane

\section{EFFICIENT PART MODELING}

Even though the above approach can be faster than meshing, the transformation of the stiffness formulation from local to global coordinates can consume a non-trivial amount of computer time. There is motivation for reducing this time. The following techniques have not been tested.

Technique \#1: Now consider a triangle bounded by one, two or three rods. This is shown in Figure (10) for three rods. These rods may be used to model shear caps. They are bundled with the membrane for the sake of computa- 
M. Blair, S. Hill, T. A. Weisshaar, R. Taylor, Rapid Modeling with Innovative Structural Concepts

tional efficiency (when the local stiffness is expanded into global stiffness).

$$
\{f\}=\left[\left[k_{M}\right]+\left[k_{S 1}\right]+\left[k_{S 2}\right]+\left[k_{S 3}\right]\right]\{\delta\}
$$

Technique \#2: One could select to save the global stiffness for each element. This could require a significant amount of computer memory. Alternatively, one could elect to recompute the global stiffness each time it is needed. When structure is removed from the overall system, one can recompute the old element stiffness matrices and subtract them from the system.

Technique \#3: The thickness $t$ of a spar can be parametrically driven along a curve $(0<\lambda<1)$ as depicted in the organic wing

$$
t(\lambda)=(1-\lambda) t(0)+\lambda t(1)
$$

Rather than gather all the stiffness into one system stiffness matrix, the stiffness of each element can be assembled into its associated spar. Subsequently, the stiffness for each spar can be assembled into the system stiffness matrix. Since the local stiffness matrix is proportional to thickness the local stiffness matrix can be transformed (expanded) to the global degrees-of-freedom in terms which are proportional to $t(0)$ and $t(1)$. Thus, the total stiffness matrix for the spar is

$$
\left[K_{P}\right]=t(0)\left[K_{P 0}\right]+t(1)\left[K_{P 1}\right]
$$

where

$$
\begin{gathered}
{\left[K_{P 0}\right]=\sum_{i=0}^{m} \frac{(1-\lambda)}{t_{i}}\left[K_{E i}\right]} \\
{\left[K_{P 1}\right]=\sum_{i=0}^{m} \frac{\lambda}{t_{i}}\left[K_{E i}\right]}
\end{gathered}
$$

Technique \#4: The thickness $t$ of a skin can be parametrically driven over a surface patch $(0<\mu<1)$ and $(0<\lambda$ $<1)$

$$
t(\mu, \lambda)=\left[\begin{array}{c}
(1-\mu)(1-\lambda) \\
(1-\mu) \lambda \\
\mu(1-\lambda) \\
\mu \lambda
\end{array}\right]^{T}\left[\begin{array}{c}
t(0,0) \\
t(0,1) \\
t(1,0) \\
t(1,1)
\end{array}\right]
$$

The total stiffness matrix for the part is

$$
\left[K_{P}\right]=\left[\begin{array}{c}
t(0,0) \\
t(0,1) \\
t(1,0) \\
t(1,1)
\end{array}\right]^{T}\left[\begin{array}{l}
{\left[K_{P 00}\right]} \\
{\left[K_{P 01}\right]} \\
{\left[K_{P 10}\right]} \\
{\left[K_{P 11}\right]}
\end{array}\right]
$$

where

$$
\left[K_{P 00}\right]=\sum_{i=0}^{m} \frac{(1-\mu)(1-\lambda)}{t_{i}}\left[K_{E i}\right]
$$

and so on

While, this element-by-element transformation procedure is not conventional, the resulting formulation is. If we had developed a conventional FEM model of a wing and substructure and subsequently constrained this resulting system of equations to the same global deformations, the identical result would have been achieved.

We pay a price for the time it takes the stiffness formulation to be developed globally. On the other hand, we gain by not having to develop a FEM mesh and the geometry it is based on. This is especially important if we are conceptualizing an organic substructure as shown in Figure (5).

We anticipate the process which we have formulated for a single wing panel could be extended to segmented wings and to fuselage structural modeling.

\section{STRUCTURAL LAYOUT}

It is important to understand the ease with which the conceptual designer will be able to lay out the skin structure and substructure. Meshing is not an issue here, thus removing a large impediment to doing structural modeling at the conceptual level.

Substructure: With a view of the nominal planform displayed, the designer interactively positions parametric curves. Each curve represents a single substructure part such as a spar. Next, a series of points are interactively selected on the curve in such a manner that they will move parametrically when the curve is moved. At each point, a normal vector is drawn which intersects the upper and lower faceted surfaces. Pairs of triangular elements are formed between the normal line segments to form segments of the substructure part. Locations of points used to form each part are maintained in a list which can be interactively altered more precisely. The material properties and thickness are maintained in another list. In this way, the designer can rapidly lay out, modify and resize the structural geometry.

Skin structure: The extent of the wing skin carries to the leading and trailing edge.

\section{STRUCTURAL CORRELATION}

Beam Model: First we simulated a straight uniform beam depicted in Figure (11). This model consisted of four ribs, three spars, and upper and lower surface skins. The beam is 300 " by 50 " by 5 " with one inch thick sur- 
M. Blair, S. Hill, T. A. Weisshaar, R. Taylor,

Rapid Modeling with Innovative Structural Concepts

faces everywhere. This was analyzed using the modeling technique described in the previous section. A tip load of 1000 pounds acted on the beam with all degrees of freedom fixed at the root.

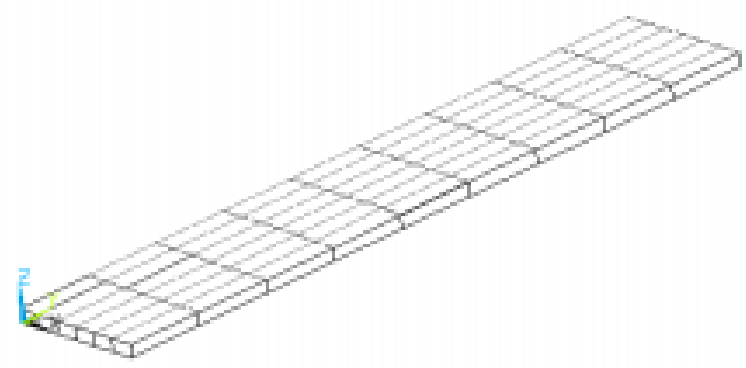

Figure (11) Test Case: The Box Beam

Beam theory predicts a tip displacement of $\delta=P L^{3} / 3 E I$. The area moment of inertia for the cross-section in Figure (11) was $664.6 \mathrm{in}^{4}$. The result is a tip displacement of 1.354 in.

ANSYS Solution: The box beam was also constructed in the ANSYS commercial FEM software. The ANSYS model used eight-node shell elements with six degreesof-freedom per node. The mesh consisting of nine elements along the length, six elements along the width and one element through the thickness. The result is a tip displacement of 1.355 in.

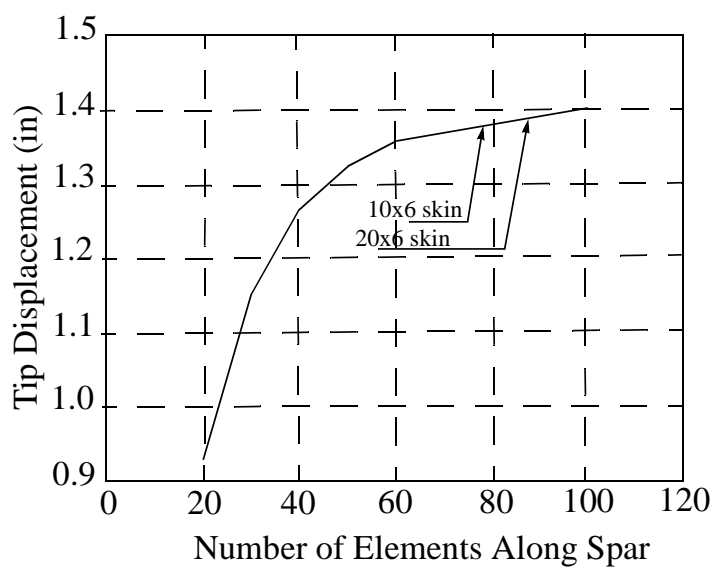

Figure (12) Box Beam Convergence Results

AML Converged Solution: Finally, the box-beam model was constructed in AML and analyzed using our modeling technique. The AML model was examined for two mesh densities. The first mesh partitioned the skin with ten elements in the span direction and six in the chord direction (10x6). The second mesh partitioned the skin with (20x6). For each skin mesh the number of spar elements was varied from 20 elements to 100 elements for each spar. (Note: as was explained earlier, the substructure mesh is independent of the skin mesh because we immediately transform local element deformations to the global deformation field.) We see the solution is approaching a converged tip displacement of $1.4 \mathrm{in}$.

In Figure (12), we see the wing skin was converged with the $(10 \times 6)$ mesh. However, we require on the order of 100 spar elements for convergence. What we learn from this is that while we require only a coarse partitioning on the skin, the substructure requires a more refined partitioning with element aspect ratio on the order of one. Lacking advanced interface element technology (Reference [9]), it is not possible to replicate this model with dissimilar meshes with standard FEM technology.

Wing Model: Next, we applied our modeling technique to a swept and tapered wing box depicted in Figure (13). The wing box model also consisted of four ribs, three spars and upper and lower surface skins. Now, however, the wing skin lay on an airfoil.

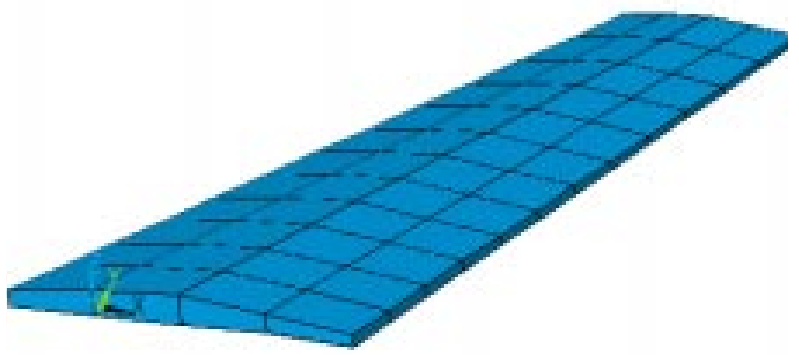

Figure (13) Test Case: The Wing Box

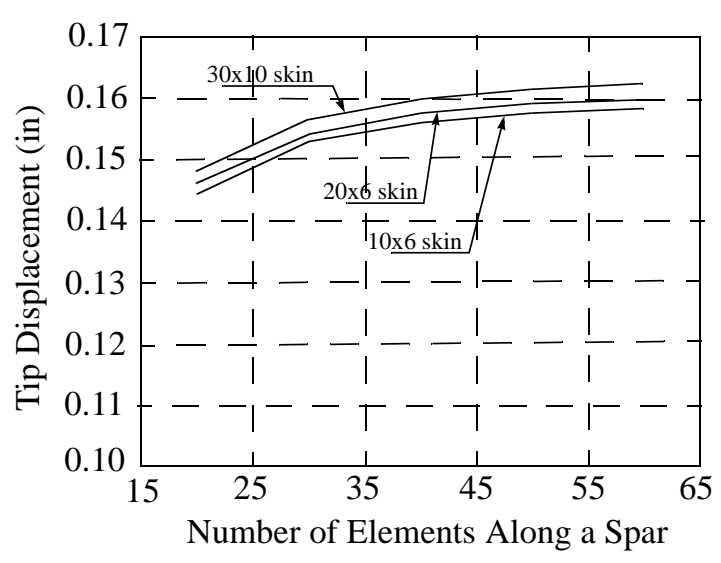

Figure (14) Wing Box Convergence Results

Again, a normal tip load was imposed on the wing with all degrees of freedom fixed at the root. 
M. Blair, S. Hill, T. A. Weisshaar, R. Taylor,

Rapid Modeling with Innovative Structural Concepts

ANSYS Solution: This model was first analyzed using ANSYS commercial FEM software. Element types and mesh densities were the same as for the box-beam model. ANSYS predicted a maximum tip displacement at the trailing edge of 0.159 .

AML Converged Solution: Next, the wing model was constructed in AML and analyzed using the same process as for the beam test case. This model converged to a maximum tip displacement at the trailing edge of approximately 0.161 inches.

\section{THE AERODYNAMIC OBJECT}

An incompressible linear vortex lattice panel object was developed entirely in AML. Since the vortex-lattice method can be simple to encode, it seemed appropriate to adopt it as our first level aerodynamic method for quick analyses.

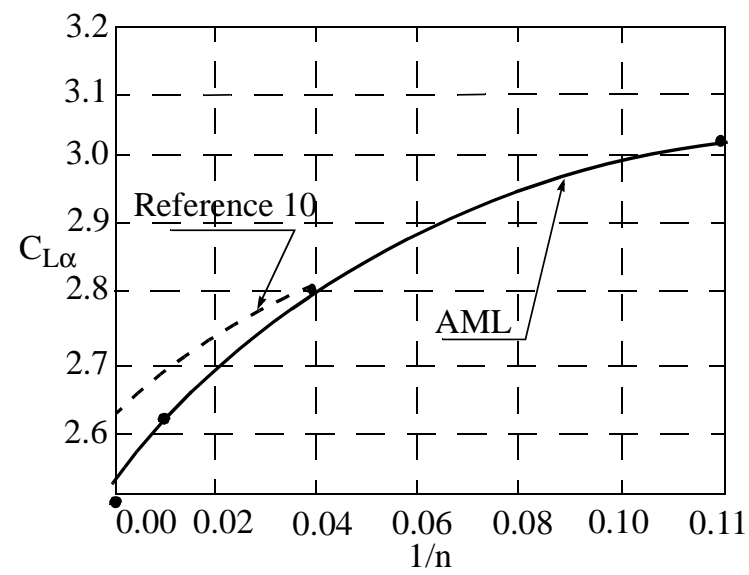

Figure (15) Convergence for AR2 Wing

In order to test the accuracy of the vortex-lattice model, we recreated a standard rectangular wing with aspect ratio of two. This is documented in Reference [10]. The results are recorded in Figure (15) with the solid curve. Three box densities were analyzed $(3 \times 3)(5 \times 5)$ and (10x10). These are plotted on the $1 / \mathrm{n}$ axis as (1/9), (1/ $25)$ and (1/100). The vertical axis is the lift curve slope. This is the lift coefficient per radian angle of attack. Results from the lattice method of Reference [10] are plotted as the dashed curve. We see a small discrepancy as the number $\mathrm{n}$ goes to infinity.

The vortex lattice elements (along with the substructure) are depicted in Figure (6). There is a vortex filament at the quarter chord of each element. The trailing wake is modeled with a flat vortex sheet in the global xy plane. The tangential flow boundary condition is enforced at the mid-span of each element at the element $3 / 4$ chord.
An interference panel is employed between the left and right panels of the wing where the fuselage is. Likewise for the tail

\section{VORTEX LATTICE APPLICATION}

The vortex-lattice object was implemented on the UCAV design depicted in Figure (6). The left and right halves of the external shape are symmetric. The geometric dimensions are provided in Table (1).

The lattice is shown on the undeformed planform in Figure (6). Each wing and tail panel was partitioned into ten elements in the spanwise direction and five elements in the chordwise direction. The flat trailing wake was extended 1000 in downstream. The vehicle was oriented at ten degrees angle of attack. The results are provided in Table (2). No aeroelastic correction has yet been implemented.

The wing structure is not symmetric. In Figure (16) we see a traditional spar/rib approach for the substructure of the right wing. We see non-traditional substructure with organic geometry on the left wing. (This departure from symmetric structural design is our artificial attempt to cram more data into one figure.) The distributed pressure load from the vortex-lattice analysis was applied directly to the wing structure. The wing tip displacements are presented in Table (3). The substructure deformations are shown in Figure (16). These are superimposed over the undeformed substructure and vortexlattice elements.

It is perhaps noteworthy that the analysis for the left wing with more spanwise substructure is more stiff than for the right wing. While this effect was to be expected, this could only be brought out with the shear deformation which is in this formulation. Equivalent plate wing analysis based on the Kirchhoff theory will not bring this out

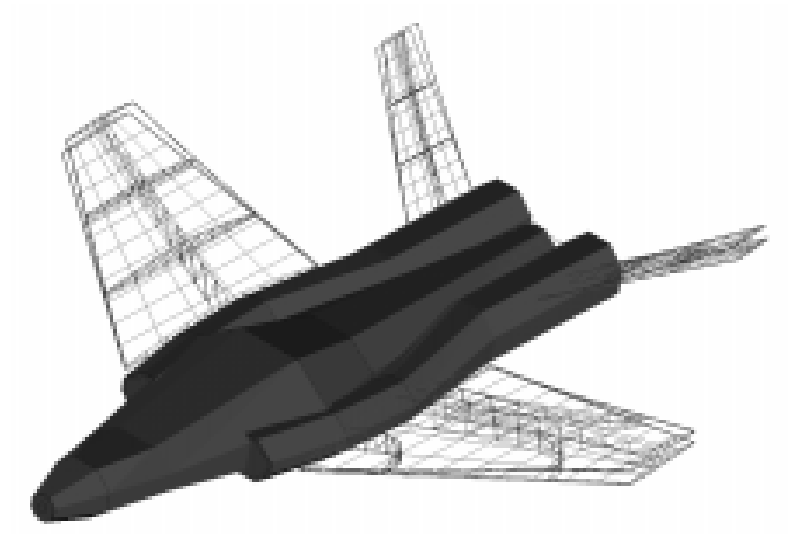

Figure (16) Deformed Wing Under Aerodynamic Load 
M. Blair, S. Hill, T. A. Weisshaar, R. Taylor,

Rapid Modeling with Innovative Structural Concepts

\begin{tabular}{|l|l|l|}
\hline \multicolumn{3}{|c|}{ Lifting Surface Geometry (right half) } \\
\multicolumn{1}{|l|}{} & Rt. Wing & Rt. Tail \\
\hline Root Coordinates for sweep axis (in) \\
\hline X(in) & 100.000 & 275.000 \\
\hline Y (in) & 56.768 & 41.929 \\
\hline Z (in) & 0.0 & 12.074 \\
\hline Sweep @ 25\% chord (deg) & 30.0 & 30.0 \\
\hline Semi-span (from root) (in) & 150.000 & 100.000 \\
\hline Dihedral (deg) & 10.0 & 30.0 \\
\hline Root chord (in) & 150.000 & 50.000 \\
\hline Tip chord (in) & 50.000 & 25.000 \\
\hline Tip twist @ 40\% chord (deg) & -5.0 & -5.0 \\
\hline Airfoil thickness (\% chord) & 10.0 & 10.0 \\
\hline Airfoil camber (\% chord) & 3.0 & 3.0 \\
\hline Structural thick (all parts) (in) & 0.05 & 0.05 \\
\hline
\end{tabular}

Looking beyond this paper, our collective experience allows us to suggest that these software innovations will allow a team of design engineers to effectively evaluate the merits of technology innovations with high fidelity data. This paper is critical in achieving this goal because it begins to establish automated dependency tracking at the peak of the systems engineering process.

\section{ACKNOWLEDGEMENTS}

A portion of this work was accomplished under STTR Phase I contract F33615-97-C-3216 “Air Vehicles Modeling and Simulation" with the Air Vehicles Directorate of the Air Force Research Laboratory.

\section{REFERENCES}

[1] Max Blair, Geetha Bharatram, Robert A. Canfield, "Designing a Blended Composite Wing and Fuselage", presented at the 6th AIAA/NASA/ISSMO Symposium on MultiDisciplinary Analysis and Optimization 4-6 September 1996 in Bellevue WA

\begin{tabular}{|l|l|}
\hline Angle of attack & $10(\mathrm{deg})$ \\
\hline Dynamic pressure & $297(\mathrm{psf})$ \\
\hline Reference area & $226\left(\mathrm{ft}^{2}\right)$ \\
\hline Lift coefficient & 0.468 \\
\hline Drag coefficient & 0.072 \\
\hline Lift & $31390(\mathrm{lbs})$ \\
\hline Drag & $4826(\mathrm{lbs})$ \\
\hline
\end{tabular}

[2] Max Blair, Steven R. LeClair, Jeffery V. Zweber and Adel Chemaly, "MultiDisciplinary Design for Uninhabited Air Vehicles", presented at the IEEE Sixth Workshop on Enabling Technologies: Infrastructure for Collaborative Enterprises, 18-20 June 1997 at Cambridge MA.

[3] Jeffery V. Zweber, Max Blair, Geetha Bharatram, Hilmi Kamhawi, "Structural and Manufacturing Analysis of a Wing Using the Adaptive Modeling Language", AIAA 98-1758 presented at the 39th AIAA/ASME/ ASCE/AHS/ASC Structures, Structural Dynamics and Materials Conference 20-23 April 1997 in Long Beach CA.

[4] D. J. Neill and D. L. Herendeen, "ASTROS Enhancements", WL-TR-96-3004 (Vol I: "ATROS User's Manual”), WL-TR-96-3005 (Vol II: “ASTROS Programmer's Manual”), WL-TR-95-3006 (Vol III: “ASTROS Theoretical Manual”).

Table (3) Wing Tip Displacement for UCAV

\section{CONCLUSIONS}

We have been successful in demonstrating the feasibility of using an advanced design modeling environment with dependency-tracking, demand-driven calculations and run-time object creation to produce a conceptual level air vehicle model with aspects of geometric modeling, aerodynamic modeling and structural modeling. While it is difficult to measure gains in design efficiency, we believe we have at least accomplished the first step in establishing the feasibility.

[5] Michael H. Shirk, Terrence J. Hertz and Terrence A. Weisshaar, "Aeroelastic Tailoring - Theory, Practice, and Promise", AIAA Journal of Aircraft, Vol 23, No 1, January 1986

[6] L. A. McCullers, R. W. Lynch, "Dynamic Characteristics of Advanced Filamentary Composite Structures, Volume II - Aeroelastic Synthesis Procedure Development”, AFFDL-TR-73-111, September 1974

[7] Gary L. Giles, "Further Generalization of an Equivalent Plate Representation for Aircraft Structural Analy- 
M. Blair, S. Hill, T. A. Weisshaar, R. Taylor,

Rapid Modeling with Innovative Structural Concepts

sis", AIAA Journal of Aircraft, Vol 26, No 1, January 1989.

[8] Gary L. Giles and Keith Norwood, "Coupling Equivalent Plate and Finite element Formulations in MultipleMethod Structural Analyses", AIAA Journal of Aircraft, Vol 31, No 5, Sept-Oct 1994.

[9] David R. Oakley, Graham S. Rhodes and Lonny B. Kruger, "Interface-Driven Multidisciplinary Design of Large-Scale Aircraft Structures" WL-TR-97-3078, February 1997.

[10] Max Blair, "A Compilation of the Mathematics Leading to the Doublet-Lattice Method" WL-TR-953022 (supersedes WL-TR-95-3022), November 1994. 